\title{
Critical Scaling of the Conductance in a Disordered Insulator
}

\author{
M. A. Paalanen, T. F. Rosenbaum, (a) G. A. Thomas, and R. N. Bhatt \\ Bell Laboratories, Murray Hill, New Jersey 07974 \\ (Received 26 August 1983)
}

\begin{abstract}
A critical scaling of the real and imaginary parts of the low-frequency ac conductance of insulating phosphorus-doped silicon near the metal-insulator transition has been observed. The results are interpreted as evidence of an electron glass, i.e., glasslike behavior, intimately connected with the scaling description of the transition, in which Coulomb interactions play a significant role.
\end{abstract}

PACS numbers: $72.15 . \mathrm{Cz}, 71.30 .+\mathrm{h}, 72.20 . \mathrm{Fr}$

The evidence of a significant role played by electron-interaction effects in disordered metals ${ }^{1-5}$ suggests an even greater role in the insulating phase $\mathrm{e}^{6,7}$ where metallic screening is absent. Historically, a number of experiments in the localized regime have been explained on the basis of the noninteracting Anderson model, in which the insulator is viewed as a Fermi glass. ${ }^{8.9}$ The most widely applied consequences of this model have been the Mott " $T^{1 / 4}$ " variable-range hopping law ${ }^{9}$ and the Austin-Mott ${ }^{10}$ low-frequency ac conductivity. These conclusions have been challenged by Efros and Shklovskii ${ }^{11}$ who claim that electron-electron interactions, no matter how small, because of their long-range $(1 / r)$ nature in the insulating phase, give rise to a soft Coulomb gap ${ }^{12}$ where the density of states goes continuously to zero at the Fermi level. While recent numerical calculations on a lattice model of this "electron glass" with strongly localized state ${ }^{13}$ confirm this, evidence from real experimental systems is somewhat scant ${ }^{11}$ and unconvincing. Our results support the Efros-Shklovskii type of model.

We have measured the complex ac conductance of phosphorus-doped silicon ( $\mathrm{Si}: \mathrm{P})$ just on the insulating side of the metal-insulator transition. Using uniaxial stress ${ }^{14}$ to tune $n_{c}$ within $0.1 \%$ of the sample density $\left(n \sim 3.6 \times 10^{18} \mathrm{~cm}^{-3}\right)$, we monitor the ac response as a function of temperature $(T)$, frequency $(\omega)$, and stress $(S)$. Our measurements span the range $10^{2} \mathrm{~Hz}<\omega / 2 \pi<10^{5} \mathrm{~Hz}$ and $10 \mathrm{mK}<T<100 \mathrm{mK}$, and are in the limit $\hbar \omega \ll k_{\mathrm{B}} T$ $\ll<\boldsymbol{R}_{\text {eff }}$ with four decades separating each inequality ( $\boldsymbol{R}_{\text {eff }}$, the P ionization energy, is $45.5 \mathrm{meV}$ $\simeq 530 \mathrm{~K} \simeq 1.1 \times 10^{13} \mathrm{~Hz}$ ). We find a sublinear dependence of the real part on $\omega\left(\sigma=K \omega^{s}, s \leqslant 1\right)$ and a corresponding infrared divergence of the dielectric constant, implying glasslike behavior. In addition, the prefactor $K$ tends to diverge as $n \rightarrow n_{c}{ }^{-}$ with the same exponent as the zero-frequency "optical" dielectric constant, ${ }^{7}$ indicating that this glassy behavior is intimately connected with the scaling description of the metal-insulator transition. At finite $T$, we observe a positive $T^{2}$ correction which also scales with the $T=0$ part.

The measurement geometry is shown in the inset of Fig. 1. After etching of the surfaces of an insulating $\mathrm{Si}: \mathrm{P}$ sample close to $n_{c}, 1000-\AA$-thick gold layers were evaporated on opposite faces, forming a capacitor with area $1.6 \mathrm{~mm}^{2}$ and thickness $0.35 \mathrm{~mm}$. The Schottky barriers, formed at the sample surfaces below the gold platings, had a capacitance $C_{B}=1100 \mathrm{pF}$ in the metallic phase. The complex conductance $Y=G+j \omega C$ was measured with a capacitance bridge. Small voltages $\left(<10^{-4} \mathrm{~V}\right)$ and power levels $\left(\sim 10^{-12} \mathrm{~W}\right)$ were

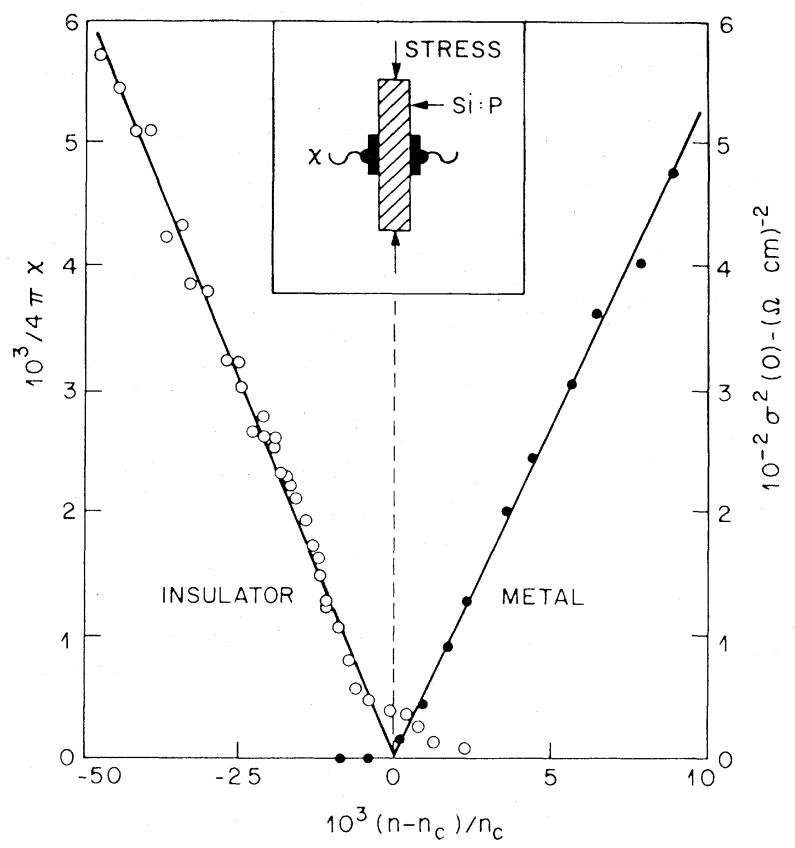

FIG. 1. Inverse donor polarizability $(4 \pi \chi)^{-1}$ at 31 $\mathrm{kHz}$ and square of the conductivity (Ref. 4), both extrapolated to $T=0$, as functions of $n-n_{c}$. Inset shows sample geometry in the capacitance-bridge measurement. 
used.

Figure 1 shows the inverse donor polarizability $(4 \pi \chi)^{-1} \equiv\left(\epsilon-\epsilon_{S i}\right)^{-1}$ at a typical frequency $(\nu=31$ $\mathrm{kHz}$ ) and extrapolated to $T=0$ (typically within $5 \%$ of the value at our lowest $T$ ) as a function of $n$ $-n_{c}$. The host dielectric constant (amounting to a background capacitance of $0.5 \mathrm{pF}$ ) has been subtracted. The linear variation implies a critical divergence $4 \pi \chi \sim\left(n_{c} / n-1\right)^{-\zeta}$ with $\zeta \approx 1.0$, consistent with the divergence of the "zero-frequency" dielectric constant obtained with high-frequency probes ${ }^{7}$ (Kramers-Kronig analysis of farinfrared absorption above $10^{11} \mathrm{~Hz}$, and microwave cavity resonance at $4 \times 10^{8} \mathrm{~Hz}$ ). Similar results are found at other frequencies studied. The rounding of the data close to the transition $(4 \pi \chi>2000)$ is due to the Schottky barriers. On the right of Fig. 1 is plotted the square of the $T$ $=0 \mathrm{dc}$ conductivity in the metallic phase from an earlier, four-probe measurement. ${ }^{5}$

In the same region, we find a variation of conductance with $\omega$ (open circles, Fig. 2) of the form $\sigma(\omega)=K \omega^{s}$ with $s=0.9 \pm 0.1$. [ However, we cannot differentiate between $\omega^{s}, \omega \ln (1 / \omega)$, or other similar forms.] Such a sublinear $\omega$ dependence of $\sigma$ has been seen ${ }^{15}$ deep in the insulating region at high $\boldsymbol{T}$, as well as in amorphous semiconductors, ${ }^{9}$ and ascribed to phonon-assisted hopping. ${ }^{10,11}$ Our experiment differs in that it is in the critical region and allows an extrapolation to $T=0 \mathrm{~K}$. Our central result is that we find a critical dependence of the prefactor $K$ with stress, which scales with the divergence of the dielectric constant:

$$
K=K_{0}\left(n_{c} / n-1\right)^{-\zeta},
$$

where $\zeta \simeq 1$ and $K_{0}$ is a constant. This is demonstrated in the inset of Fig. 2 which shows a linear relationship between the real and imaginary part of the conductance as the two are varied by the application of stress.

The sublinear dependence of $\sigma$ on $\omega$ implies, via a Kramers-Kronig relationship, a weak (logarithmic, or small-power law) divergence of the dielectric constant $\epsilon(\omega)$ as $\omega \rightarrow 0$. This is consis-

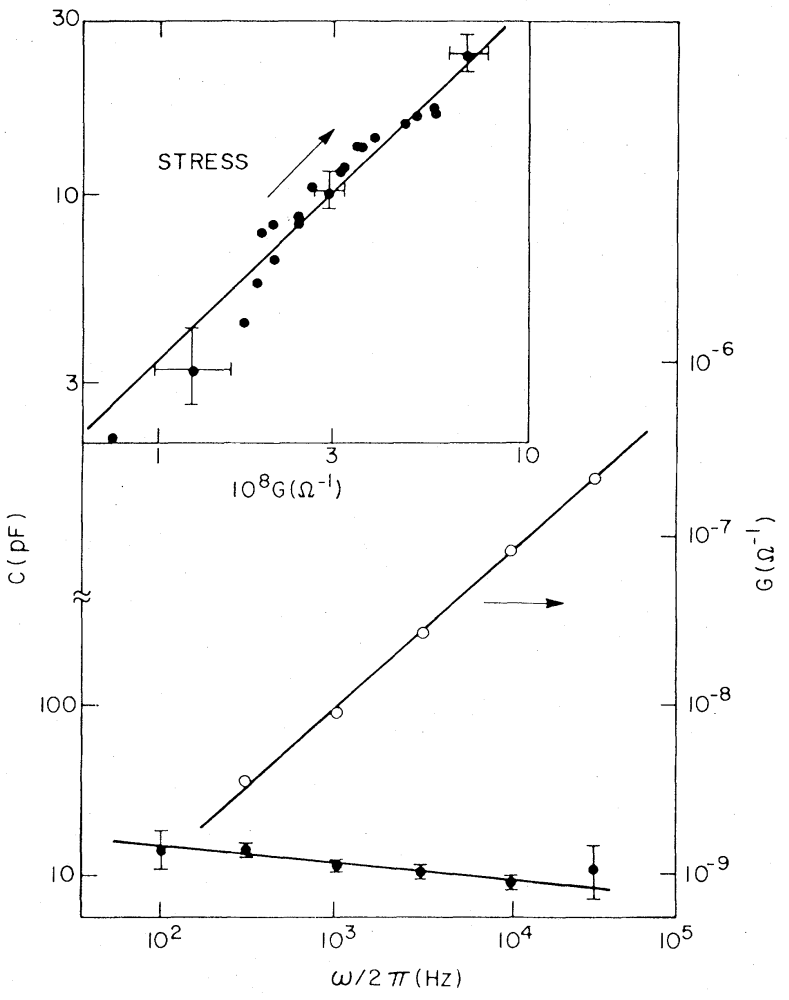

FIG. 2. Variation of conductance $G$ (open circles) and donor capacitance $C$ (solid circles) with frequency $\omega / 2 \pi$ at $T=13 \mathrm{mK}$ at a typical stress $[S=1.72 \mathrm{kbar}$, $4 \pi \chi(0) \simeq 180$ ]. Solid lines are fits by the forms $\omega^{s}$ and $\omega^{s-1}$ respectively, with $s=0.9 \pm 0.1$ (data also consistent with other forms, see text). Inset shows proportionality between real and imaginary parts of the conductance (extrapolated to $T=0 \mathrm{~K}$ ) at $31 \mathrm{kHz}$ as $n \rightarrow n_{c}{ }^{-}$.

tent with the rise of the capacitance seen at low frequency (solid circles, Fig. 2) and indicative of glassy behavior. As $n \rightarrow n_{c}{ }^{-}$, the variation with $\omega$ increases proportionally with the absolute value at a given $\omega$.

If we take a hopping conductivity $\sigma(\omega)=K \omega^{s}$, with $s<1$ for $0<\omega<\omega_{c}\left(\hbar \omega_{c}\right.$ is a cutoff $\sim k_{\mathrm{B}} T$ above which the hopping conductivity joins onto the optical conductivity ${ }^{7}$ ), the dielectric constant is given by

$$
\epsilon(\omega)=8 \int_{0}^{\infty} \frac{\sigma\left(\omega^{\prime}\right) d \omega^{\prime}}{\omega^{\prime} 2-\omega^{2}}=8 K \omega^{s-1}\left[\frac{\pi}{2} \tan \frac{\pi s}{2}-\frac{\left(\omega / \omega_{c}\right)^{1-s}}{1-s}\right]+\epsilon_{\mathrm{op}}+\epsilon_{\mathrm{Si}},
$$

where $\epsilon_{S i}(=11.4)$ is the Si dielectric constant and $\epsilon_{\text {op }}$ that coming from the donor optical $\left(\omega>\omega_{c}\right)$ conductivity, whose weak $\omega$ dependence has been neglected. The first two terms represent the (frequencydependent) donor polarizability, $4 \pi \chi(\omega)$. The ratio of the imaginary and real parts of the donor conductance is thus

$$
\frac{\omega C(\omega)}{G(\omega)} \equiv \frac{\omega \chi(\omega)}{\sigma(\omega)}=\tan \frac{\pi s}{2}-\frac{2\left(\omega / \omega_{c}\right)^{1-s}}{\pi(1-s)}+\frac{\epsilon_{\mathrm{op}} \omega^{1-s}}{4 \pi K}
$$


Because $1-s \approx 0.1$ is small, the last two terms make significant contributions even at the low $\omega$ of the present experiment (the variation of $\omega C / G$ with $\omega$ is weak for the same reason). However, Eq. (3) demonstrates that if $\omega C / G$ is independent of stress, the prefactor $K$ of the "hopping" conductivity must scale with the dielectric constant $\epsilon_{\mathrm{op}}$ as a result of the optical conductivity, i.e., both must diverge similarly as $n \rightarrow n_{c}{ }^{-}$(Fig. 2 inset shows this effect).

While both the noninteracting Fermi glass ${ }^{10}$ and interacting-electron glass ${ }^{11}$ models give a hopping conductivity linear in $\omega$ with logarithmic corrections, their implications in the critical region are very different. In the noninteracting case, $K \propto \xi^{5}$ where $\xi$ is the localization length $\left[\xi \sim\left(n_{c} / n\right.\right.$ $\left.-1)^{-\nu}\right]$, whereas ${ }^{7} \epsilon_{\text {op }} \propto \xi^{2}$. This implies a variation in $\omega C / G$ with $S$ [see Eq. (3)], in contrast to our experimental results. For the Efros-Shklovskil electron glass, however, $K=c_{0} \omega \epsilon_{\mathrm{op}} \ln ^{-1}\left(\omega_{c} /\right.$ $\omega)$ for $\omega \ll \omega_{c}$ in the critical region, ${ }^{16}$ where $c_{0} \sim 1$. Thus $K \propto \epsilon_{\text {op }}\left(\ln \omega_{c}\right.$ does not vary rapidly $\left.{ }^{16}\right)$, in agreement with our experiment; further, the magnitude also agrees to within a factor of 2 .

We find a strong temperature dependence of both the real and imaginary parts of the conductance. Figure 3 show $s$ the capacitance $C\left(=C_{\text {meas }}\right.$ $-0.5 \mathrm{pF}$ ) for different $S$ values at $31 \mathrm{kHz}$ as a function of $T^{2}$. The solid-line fits imply a $T$ variation of the form $C(T)=C(0)\left[1+\left(T / \Delta_{C}\right)^{2}\right]$. A similar dependence is found for the conductance, $\boldsymbol{G}(T)=G(0)\left[1+\left(T / \Delta_{G}\right)^{2}\right]$, at the frequencies studied. The inset shows the variation of the coefficient of the $T^{2}$ part of the donor polarizability [or $C(T)$ ] with the extrapolated $T=0$ polarizability, on a log-log plot. The straight line drawn through the data has a slope of 1.3 \pm 0.2 ; a similar plot for $G(T)$ yields a slope of $1.0 \pm 0.2$. Thus, $\Delta_{G}$ and $\Delta_{C}$ do not appear to vary critically at $n_{c}$ [ or have very small exponents, $\sim 0.15 \pm 0.2]$. This is in contrast to the prediction of current versions of the electron-glass model where the $T$ variation of the hopping conductivity ${ }^{16}$ is not purely quadratic, and further the scale for its variation is the soft Coulomb gap, $\Delta$, which varies critically near $n_{c}$, as $\epsilon_{\mathrm{op}}{ }^{-3 / 2}$. The magnitude of $\Delta_{C}$ and $\Delta_{G}$ obtained from fitting the data is $\approx 30 \mathrm{mK}$, which agrees with theoretical estimates for $\epsilon_{\mathrm{op}} \sim 1000$, but is lower by almost two orders of magnitude for $\epsilon_{\mathrm{op}} \sim 50$. The noninteracting Fermi glass model, ${ }^{10}$ as stated earlier, gives a linear dependence on $\boldsymbol{T}$ (unlike the $T^{2}$ variation observed) and has no energy on the 30 $\mathrm{mK}$ scale.

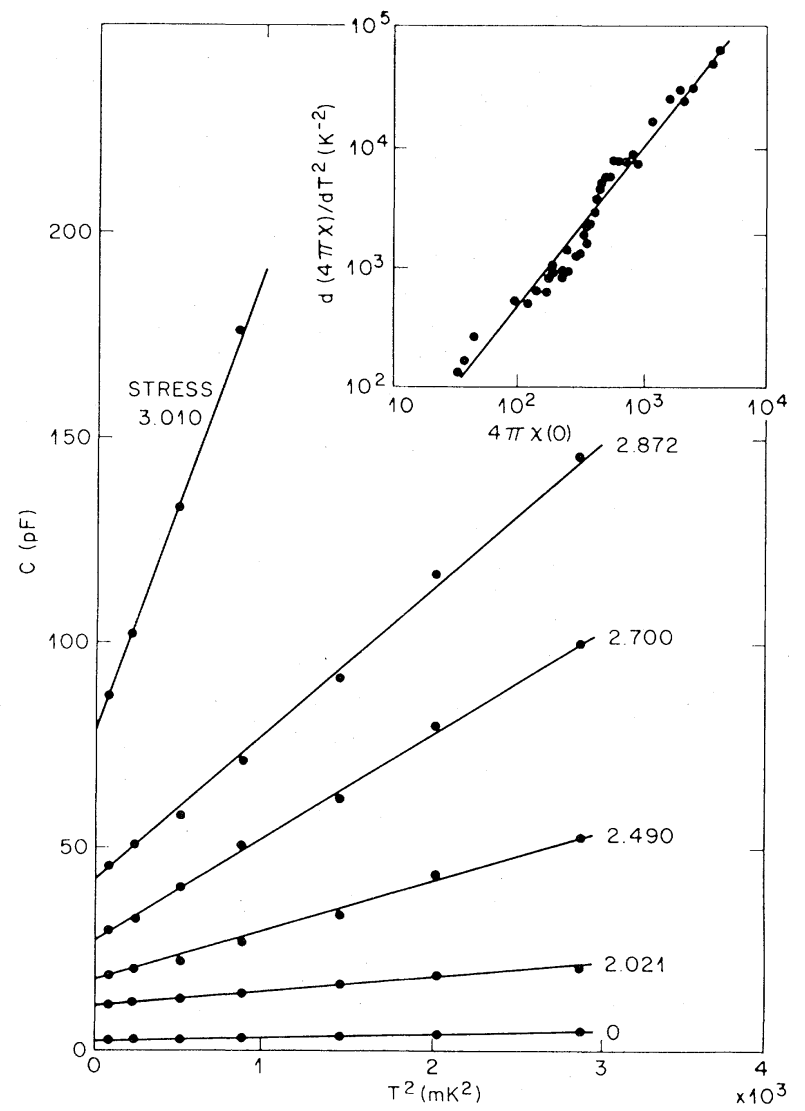

FIG. 3. Capacitance $C(T)$ at $31 \mathrm{kHz}$ in the range 10 $\mathrm{mK}<T<55 \mathrm{mK}$ at different stresses in (kilobars). Straight-line fits indicate a $T^{2}$ variation, also found for $G(T)$. Inset: a $\log -\log$ plot of the slope $d(4 \pi \chi) / d T^{2}$ with the $T=0$ polarizability, $4 \pi \chi(0)$. Straight line has slope of 1.3.

We find $\Delta_{G}$ somewhat smaller than $\Delta_{C}($ by $\approx 25 \%)$, consistent with the expectation that the $T$ dependence of $C$ comes only from the contribution due to the hopping conductivity. The difference also provides confirmation of our earlier statement that $\epsilon_{\mathrm{op}}$ is a significant portion of the total dielectric constant [ Eq. (3)].

In conclusion, we find that the low-frequency $\left(\omega \ll k_{\mathrm{B}} T\right)$ ac conductivity in $\mathrm{Si}: \mathrm{P}$ just on the insulating side of the transition varies almost linearly with $\omega$ over three decades, implying a weak, near logarithmic, infrared divergence of the dielectric function reminiscent of relaxation phenomena in glasses. Further, the prefactor appears to diverge as $n \rightarrow n_{c}{ }^{-}$with the same exponent as the optical dielectric constant. This scaling relationship is implied by the EfrosShklovskiǐ interacting-electron glass picture, but there is a discrepancy in the temperature depen- 
dence. Nevertheless, the data appear to rule out the noninteracting Fermi glass model, and point to the importance of Coulomb interactions in the description of the metal-insulator transition in $\mathrm{Si}: \mathrm{P}$.

We would like to thank P. W. Anderson, J. J. Hauser, P. A. Lee, and T. V. Ramakrishnan for helpful discussions.

(a) Present address: Department of Physics, University of Chicago, Chicago, ㅍll. 60637.

${ }^{1}$ B. L. Al'tshuler and A. G. Aronov, Solid State Commun. 30, 115 (1979), and Zh. Eksp. Teor. Fiz. 50, 2028 (1979) [Sov. Phys. JETP 50, 968 (1979)].

${ }^{2}$ R. C. Dynes and J. P. Garno, Phys. Rev. Lett. $\underline{46}$, 137 (1981).

${ }^{3}$ W. L. McMillan and J. Mochel, Phys. Rev. Lett. $\underline{46}$, 556 (1981).

${ }^{4}$ G. Hertel, D. J. Bishop, E. G. Spencer, J. M. Rowell, and R. C. Dynes, Phys. Rev. Lett. 50, 743 (1983).

${ }^{5}$ T. F. Rosenbaum, K. Andres, G. A. Thomas, and P. A. Lee, Phys. Rev. Lett. 46, 568 (1981); G. A. Thomas, M. A. Paalanen, and T. F. Rosenbaum, Phys. Rev. B 27, 3897 (1983).

${ }^{6}$ T. G. Castner, N. K. Lee, C. S. Cieloszyk, and G. L. Salinger, Phys. Rev. Lett. 34, 1625 (1975).

${ }^{7}$ M. Capizzi, G. A. Thomas, F. DeRosa, R. N. Bhatt, and T. M. Rice, Phys. Rev. Lett. 44, 1019 (1980);

T. F. Rosenbaum, K. Andres, G. A. Thomas, and R. N. Bhatt, Phys. Rev. Lett. 45, 1723 (1980); H. F. Hess, K. DeConde, T. F. Rosenbaum, and G. A. Thomas, Phys. Rev. B 25, 5578 (1982).

${ }^{8} \mathrm{P}$. W. Anderson, Comments Solid State Phys. 2, 193 (1970); L. Fleishman, D. C. Licciardello, and P. W. Anderson, Phys. Rev. Lett. $\underline{40}$, 1340 (1978).

${ }^{9}$ N. F. Mott, Philos. Mag. $\underline{19}, 835$ (1969); N. F. Mott and E. A. Davis, Electronic Processes in Non-Crystalline Materials (Oxford Univ. Press, London, 1979).

${ }^{10}$ I. G. Austin and N. F. Mott, Adv. Phys. 18, 41 (1969). ${ }^{11}$ A. L. Efros and B. I. Shklovskiŭ, J. Phys. C $\underline{8}$, L49 (1975); A. L. Efros, J. Phys. C $\underline{9}, 2021$ (1976), and Philos. Mag. 43, 829 (1981); B. I. Shklovskili and A. L. Efros, Zh. Eksp. Teor. Fiz. 81, 406 (1981) [Sov. Phys. JETP 54, 218 (1981)].

${ }^{12}$ M. Pollak, Discuss. Faraday Soc. $\underline{50}, 13$ (1970); Proc. Roy. Soc. London Ser. A 325, 383 (1971). See also W. L. McMillan, Phys. Rev. B 24, 2739 (1981). ${ }^{13}$ S. D. Baranovski ̌, B. I. Shklovskiǐ, and A. L. Efros, Zh. Eksp. Teor. Fiz. 51, 395 (1980) [Sov. Phys. JETP 51, 199 (1980)]; J. H. Davies, P. A. Lee, and T. M. Rice, Phys. Rev. Lett. $\underline{49}, 758$ (1982).

${ }^{14}$ M. A. Paalanen, T. F. Rosenbaum, G. A. Thomas, and R. N. Bhatt, Phys. Rev. Lett. 48, 1284 (1982);

R. N. Bhatt, Phys. Rev. B 26, 1082 (1982).

${ }^{15}$ M. Pollak and T. H. Geballe, Phys. Rev. 122, 1742 (1961).

${ }^{16}$ R. N. Bhatt and T. V. Ramakrishnan, private communication. 